\title{
Vibration Signature of Roller Bearing's Faults
}

\author{
Ahmed H. Osman, Assistant Professor \\ Ahmed A. Salman, Associate Professor \\ Khaled M. Fawzy, Master Student \\ Mechanical Power Engineering Department, Faculty of Engineering \\ Zagazig University, Zagazig, Egypt
}

Doi: 10.19044/esj.2019.v15n12p425 URL:http://dx.doi.org/10.19044/esj.2019.v15n12p425

\begin{abstract}
Centrifugal pumps are widely used in many industrial applications. Defective pumps cause higher rate of energy loss with related overall performance degradation. Mechanical components fail due to decrease in pump's strength or an increase in the force acting on it. Rolling bearings are one of the major components of the centrifugal pumps. Bearings are critical mechanical components in industrial machines. Bearing failure is often attributed to be one of the major causes of breakdown. Early detection of undesired conditions during the operation of the centrifugal pump has become very important in order to avoid consequential damages. Early detection is also vital for increasing availability and reliability. Automated health monitoring can reduce the outage time and repair costs. Using vibration analysis technique to monitor and evaluate the health of the machines has become an important technique in this field. In this research, we studied the vibration signatures of healthy and faulty conditions of the bearings of a pumping system. The pumping system consists of centrifugal pump and electrical motor. The vibration signatures analyze the bearing status with respect to the normal operation. The paper shows how to identify if there is a problem in the outer race of the Bearing Pass Frequency Outer Race (BPFO).
\end{abstract}

Keywords: Vibration signature, Roller Bearing, Vibration Analysis, Centrifugal Pump

\section{Introduction}

In industrial applications, ball bearings are critical mechanical components and a defection in such component would cause malfunction and may even lead to catastrophic failure of the machinery. Therefore, the condition monitoring of rolling bearings is important. In the last two decades, different methods have been used such as vibration analysis, acoustic 
measurements, and temperature measurements. Among these methods, vibration measurements and analysis are widely applied. Consequently, Mathew and Alferdson (1984) built up the procedure of condition monitoring for rolling element bearings by using vibration analysis. The vibration statistics were analyzed and several parameters (Peak Level, RMS Level, Matched Filter RMS, and Kurtosis Factor) have been assessed with reference to their effectiveness in the detection of bearing circumstance. Their conclusion was that each of the parameters has some value contingent upon the kind of bearing failure encountered. Frequency domain parameters were more predictable in the recognition of harm than time domain parameters. Tandon and Choudhury (1997) displayed associate degree analytical model for the vibration reaction of rolling element bearings because of the localized defect. They studied the amplitudes of the serious frequency parts, outer race, the inner race, and one of the rolling elements under axial and radial loads. It predicts the impact of load and pulse form on the vibration amplitude. The instance introduced here predicts a frequency spectrum. This has peaks at characteristic defect frequencies together with a modulation into the outer race rolling elements defect and inner race defect under a radial load. Tandon and Choudhury (1998) introduced an analytical model to conjecture the vibration reactions of rolling bearings because of distributed defects under radial load. Amarnath et al. (2004) built up the method to provide earlier data about progressing malfunctions. This was done to reveal the circumstance of antifriction bearings and to recognize the main points of the severity of defects before they lead to serious catastrophic failure. Many techniques can recognize the different defects in bearings such as time domain analysis, frequency domain analysis, and spike energy analysis. The outcomes of each one of these methods proved that it has the capability to investigate the bearings problems. The frequency spectrum and severity identify the exact nature of defects. Zeki Kural and Hira Karagulle (2005) studied the defect detection in rolling element bearings structure utilizing the time and frequency domain parameters based on the finite element vibration analysis. They found that the defect position on the outer ring, number of defects and defect locations, on the inner ring or on the rolling element, and the shaft speed affect the statistical indices. Furthermore, the envelope method is effectively used to detect the outer and inner ring defects, but it is not easy to detect rolling element defects via envelope and band ratio. Shinde et al. (2014) concentrated on the detection of a localized defect in a double row ball bearing. The frequency domain approach was used followed by experimentation for the detection of vibration signal in which single point defects are artificially created on the ball, the inner race, and outer race. The data clarify that the amplitude increases together with increasing the speed and the detect size and, however, it decreases with growing load. They used 
the Frequency Surface Response (FSR) to estimate the amplitudes. They found that there is a good agreement between the experimental and estimated values. Brian Graney and Ken Starry (2012) presented a paper on Rolling Element Bearing Analysis. This paper is focused on bearing failure, which occurs randomly due to its wear. The dynamic forces and lubrication problem may accelerate this wear. Ravindra Tarle et al. (2015) presented a project to address design, experimentation, and validation analysis of fault diagnosis of ball bearing related to rotor system. They presented a detail analysis using FFT methodology to find out the possible faults and finally validate with MATLAB software. They identified the faults on a single rotor test rig. In addition to this, they applied the lubrication analysis by using FFT analyzer for bearing with lack of lubrication and bearing with lubrication. Ebrahim Ebrahimi (2012) presented an experimental study regarding the good shape of ball bearings and localized defect in the outer race ball bearings under different levels of faults, various load, and speed conditions. Sharma et al. (2011) carried out an experimental study of a rotor-bearing system to obtain the vibration response due to localized defects such as spall on outer race, inner race, and roller. The results of vibration responses showed that every defect excites the system at its characteristic frequency. Response surface methodology also shows that the severe vibration (Max. peak of vibration excitation) occurs in case of bearing with outer race defect. In addition, interaction of outer and inner race defect produces severe vibrations. A defect in roller has less effect on the amplitude of vibration.

\section{Rolling Bearings Elements}

A rolling element bearing consist of the outer race, cage, the inner race, and rolling elements. Any part of the bearing can be defected and cause high-frequency vibrations. In many cases, it is probably to recognize the bearing component that defects because of excited specific vibration frequencies. It is easy to detect the raceways and rolling elements defects but the crop up in bearing cages cannot be detected easily. Although many methods are available to detect the position of the defect, there are no established methods to expect when the bearing defect occur and turn into a functional failure. The frequencies of the defect bearing are not integrally harmonic with the running speed. There is also commercial software that has a bearing database available that readily provides the values upon entering the requisite bearing number. However, to detect the bearing defect frequencies, the following formulas are used.

$$
B P F I=\frac{N b}{2}\left(1+\frac{B d}{P d} \cos (\theta)\right) r p m
$$




$$
\begin{gathered}
B P F O=\frac{N b}{2}\left(1-\frac{B d}{P d} \cos (\theta)\right) r p m \\
F T F=\frac{1}{2}\left(1-\frac{B d}{P d} \cos (\theta)\right) r p m \\
B S F=\frac{P d}{2 B d}\left(1-\left(\frac{B d}{P d}\right)^{2}(\cos (\theta))^{2}\right) r p m
\end{gathered}
$$

Bearing damage progresses through four stages: The first stage is just high-frequency vibration. At the second stage, the resonance frequencies of the bearing are observed. Discrete frequencies can be seen at the third stage. At the fourth stage, a high-frequency random noise that keeps broadening and rising in average amplitude with increased fault severity will be observed.

\section{Stage 1 of Bearing Defect}

The FFT spectrum for bearing defects can be split into four zones (A, B, C, and D). These zones are described as:

Zone A: Harmonics zone and machine rpm

Zone B: Bearing defect frequencies zone (5000-30000 CPM)

Zone C: Bearing component natural frequencies zone (30000-120000 CPM)

Zone D: High-Frequency-Detection (HFD) zone (beyond 120000 CPM).

The first indications of bearing wear show up in the ultrasonic frequency which ranges from approximately $20-60 \mathrm{kHz}(120000-360000$ CPM). As shown in Figure 1, there is no visible defects in the bearing raceways or rolling elements during the first stage but the shine of a new bearing for the raceway no longer exist and may appear dull gray (Paresh Girdha, 2004).
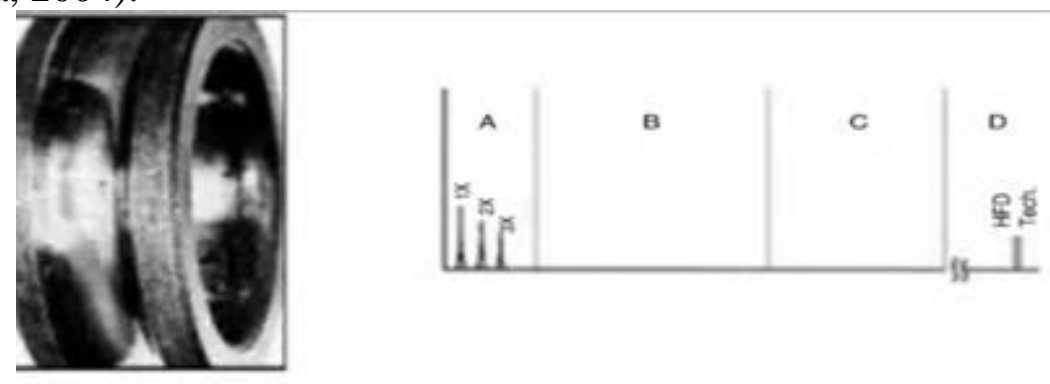

Figure 1. Small defects in the raceways of a bearing 


\section{Stage 2 of Bearing Defect}

In the following stage, as shown in Figure 2, minute pits begin to develop due to the fatigued raceways. During passing, the rolling elements over these pits and the ringing or the bearing component natural frequencies are generated that predominantly occur in the range of 30000-1200000 CPM range. At the end of stage two, it is possible that the sideband frequencies (bearing defect frequency $\pm \mathrm{rpm}$ ) appear above and below the natural frequency peak depending on the severity (Paresh Girdha, 2004).

\section{Stage 3 of Bearing Defect}

In the third stage, as shown in Figure 3, the discrete bearing frequencies and harmonics are visible, and this may appear with several sidebands. Wear is usually now visible on the bearing and may expand through to the edge of the bearing raceway. The earlier stage minute pits are now developed into bigger pits and their numbers also increase. Thus, it is usually advisable for the bearing at this stage to be replaced.
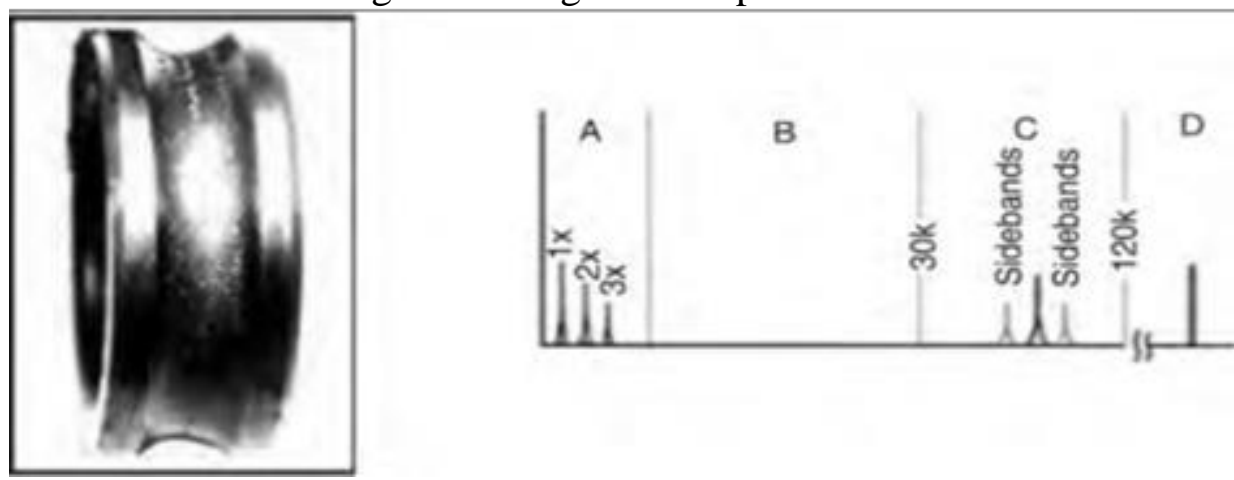

Figure 2. More obvious wear in the form of pits
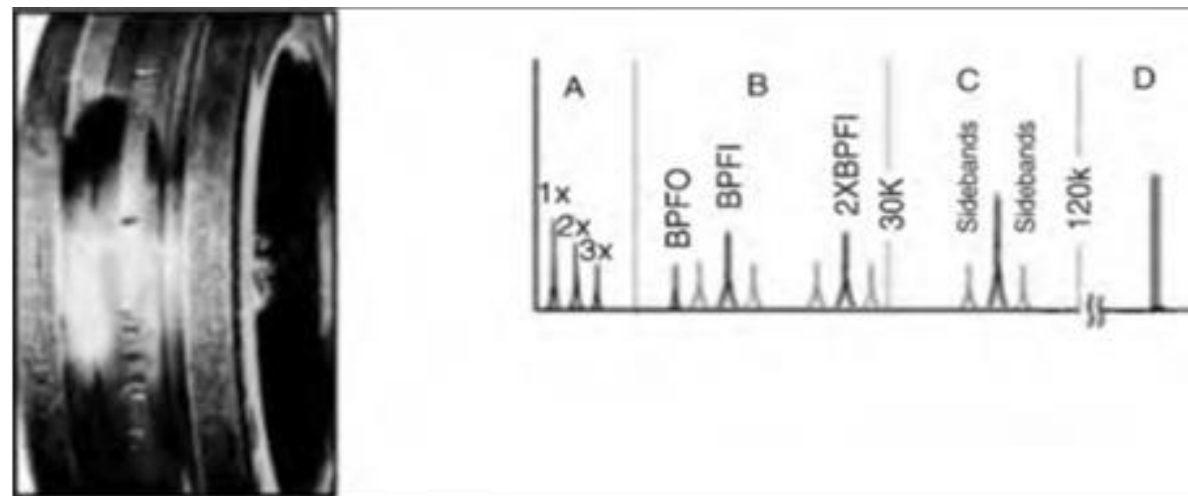

Figure 3. Wear is now clearly visible over the breadth of the bearing 


\section{Stage 4 of Bearing Defect}

In the last stage, as shown in Figure 4, the pits converge with each other, developing rough tracks and spelling of the bearing raceways or/and rolling elements. The bearing is among extremely harmed circumstance now. Even the amplitude of the $1 \mathrm{X}$ rpm component will rise. In addition, many running speed harmonics will increase as $1 \mathrm{X} \mathrm{rpm}$ increase. It will cause the rotor displacement to be higher as the higher clearance in the bearing. Bearing component natural frequencies and discrete bearing defect frequencies begin to merge into a random and a broadband high-frequency 'noise floor'. Initially, as the broad noise is large, average amplitude may happen. However, the noise width will increase, and the average amplitude will drop. Finally, the amplitude will rise again and the span of the noise floor increases (Paresh Girdha, 2004).

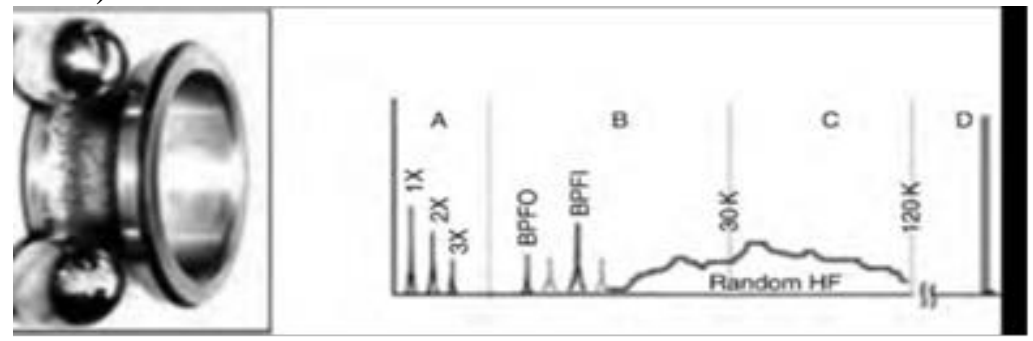

Figure 4. Severely damaged bearing at the final stage of wear

However, amplitudes of the high-frequency noise floor and some of the HFD may decrease (due to pits flattening to become spalls). Nevertheless, prior to failure spike, energy will usually grow to extreme amplitudes. By this time, the bearing will make a lot of noise and generate excessive vibration and it will become hot. If it can run further, the rolling elements will go loose, and the cage will break. Under the bearing area, there will be serious damage to the shaft area after the bearing elements run into each other, tuning, twisting, and welded to each other making the machine to trip on overload (ISO 108167).

\section{Data Acquisition System}

Centrifugal pump (seawater pump 1029A Figure 5) consists of the electric motor as a prime mover connected to the pump with fixable coupling. The pump consists of four stages. The motor speed is $2900 \mathrm{rpm}$ and its rated power is 132 kilowatts. This pumping system uses sea water. In this case, the pump was investigated due to an alarming abnormal sound. The inspection showed that the source of abnormal noise was from the motor. Two types of bearings (SKF 7309 and SKF 6308) support the pump shaft. Two bearings (SKF 6208) support the motor shaft. The monitoring system consists of (Data Collector/Analyzer) analyzer type DLI Watchman. DCA-31 ${ }^{\mathrm{TM} B}$ was coupled 
with Expert ALERTTM software with two-channel configurations. The accelerometer type ICP (Integrated Circuit Piezoelectric) is piezoelectric. The accelerometer measures the vibration signals for three directions (axial, horizontal and vertical) at four points for the pump and motor. The schematic diagram of the pump system and the location of the accelerometer are shown in Figure 6. The accelerometers are arranged in the following order: motor NDE 1, motor DE 2, pump DE 3, and pump NDE 4. The accelerometer is mounted using a magnet and positioned perpendicular to the alternator bearing housing.

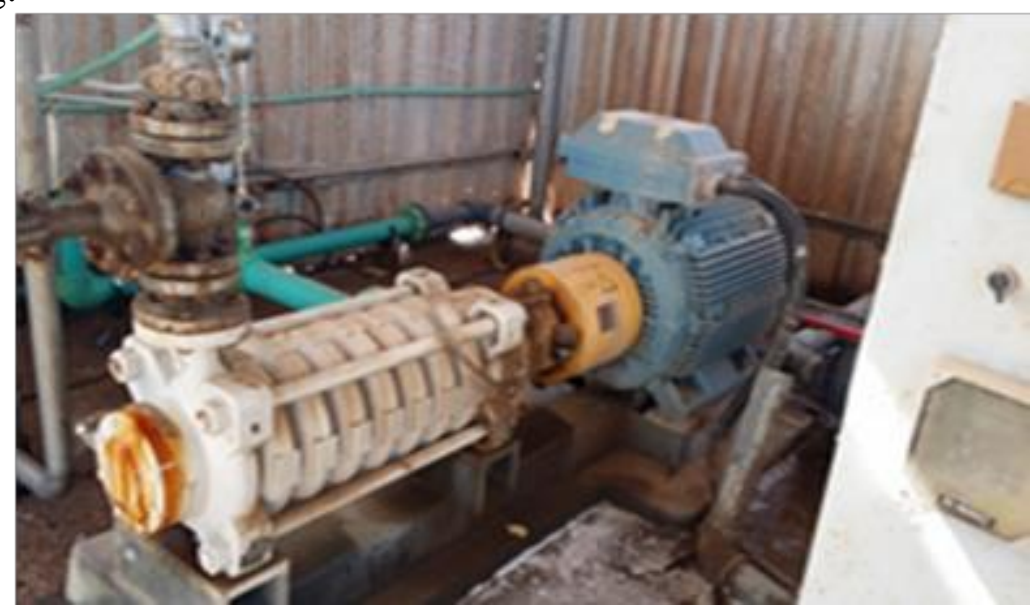

Figure 5. Sea water pump 1029 A

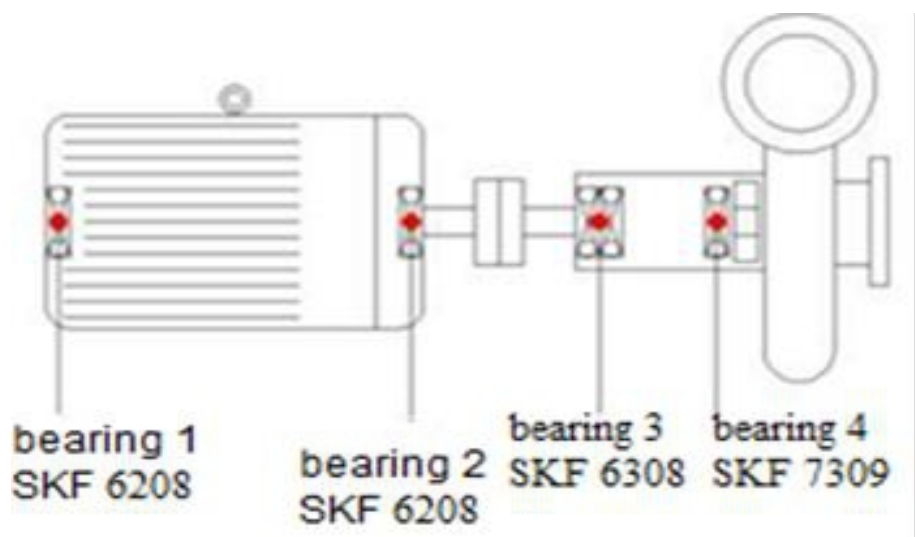

Figure 6. The point of measurement at the four bearing's locations

\section{Bearing Specifications}

The equations 1 to 4 calculates the bearing frequencies. The summary of these calculations is shown on Table 1. Overall vibration levels are measured and analyzed at both the normal and faulty conditions on different dates. The results are compared with ISO10816-7. The defects are also 
identified through vibration analysis by finding the difference in amplitudes.

Table 1. The Frequency of the Faulty Bearings' Components

\begin{tabular}{llll} 
Bearing \# & SKF 6208 & SKF 7309 & SKF 6308 \\
\hline No. of balls NB & 9 & 12 & 8 \\
\hline Ball diameter (mm) BD & 4.68 & 6.87 & 5.93 \\
\hline Pitch diameter (mm)PD & 23.62 & 28.54 & 25.59 \\
\hline Pitch angle, $\theta$ & 0 & 30 & 0 \\
\hline BPFI & $5.39 \mathrm{X}$ & $7.25 \mathrm{X}$ & $4.93 \mathrm{X}$ \\
\hline BPFO & $3.61 \mathrm{X}$ & $4.75 \mathrm{X}$ & $3.07 \mathrm{X}$ \\
\hline FTF & $0.40 \mathrm{X}$ & $0.40 \mathrm{X}$ & $0.38 \mathrm{X}$ \\
\hline BSF & $2.42 \mathrm{X}$ & $1.99 \mathrm{X}$ & $2.04 \mathrm{X}$
\end{tabular}

\section{Measurements \& Discussion}

Normal Operation

The frequency spectrums of the healthy pump were recorded. Consequently, it can be used as baseline data for future analysis. This spectrum is shown in the Figures 7, 8, and 9. The signals are measured at three directions (axial, vertical and horizontal) for the pump's bearings and motor's bearings. The graphs show normal overall reading for all bearings. Table 2 shows the normal readings compared with alarm levels of the pump and motor at the four positions of the pumping system (Motor NDE, Motor DE, Pump DE and Pump $\mathrm{NDE})$ which is at the three axes (V, H, A). It can be seen that these readings are normal.

Table 2. The normal overall reading

\begin{tabular}{|c|c|c|c|c|c|c|c|c|c|c|c|c|c|c|}
\hline & \multicolumn{3}{|c|}{ Motor NDE } & \multicolumn{4}{c|}{ Motor DE } & \multicolumn{4}{c|}{ Pump DE } & \multicolumn{3}{c|}{ Pump NDE } \\
\hline & $\mathrm{V}$ & $\mathrm{H}$ & $\mathrm{A}$ & $\mathrm{V}$ & $\mathrm{H}$ & $\mathrm{A}$ & $\mathrm{V}$ & $\mathrm{H}$ & $\mathrm{A}$ & $\mathrm{V}$ & $\mathrm{H}$ & $\mathrm{A}$ \\
\hline Unit & \multicolumn{9}{|c|}{ mm/s RMS } \\
\hline Alarm & 4.5 & 4.5 & 4.5 & 4.5 & 4.5 & 4.5 & 4.5 & 4.5 & 4.5 & 4.5 & 4.5 & 4.5 \\
\hline Critical & 7 & 7 & 7 & 7 & 7 & 7 & 7 & 7 & 7 & 7 & 7 & 7 \\
\hline $\begin{array}{c}\text { Normal } \\
\text { Reading } \\
\text { s }\end{array}$ & 1.9 & 1.38 & 1.25 & 1.77 & 2.06 & 1.19 & 1.42 & 0.904 & 1.42 & 1.18 & 0.942 & 1.02 \\
\hline Faulty & 3.42 & 3.55 & 1.69 & 2.84 & 2.68 & 1.54 & 2.02 & 1.84 & 2.66 & 3.86 & 3.44 & 11.91 \\
\hline
\end{tabular}




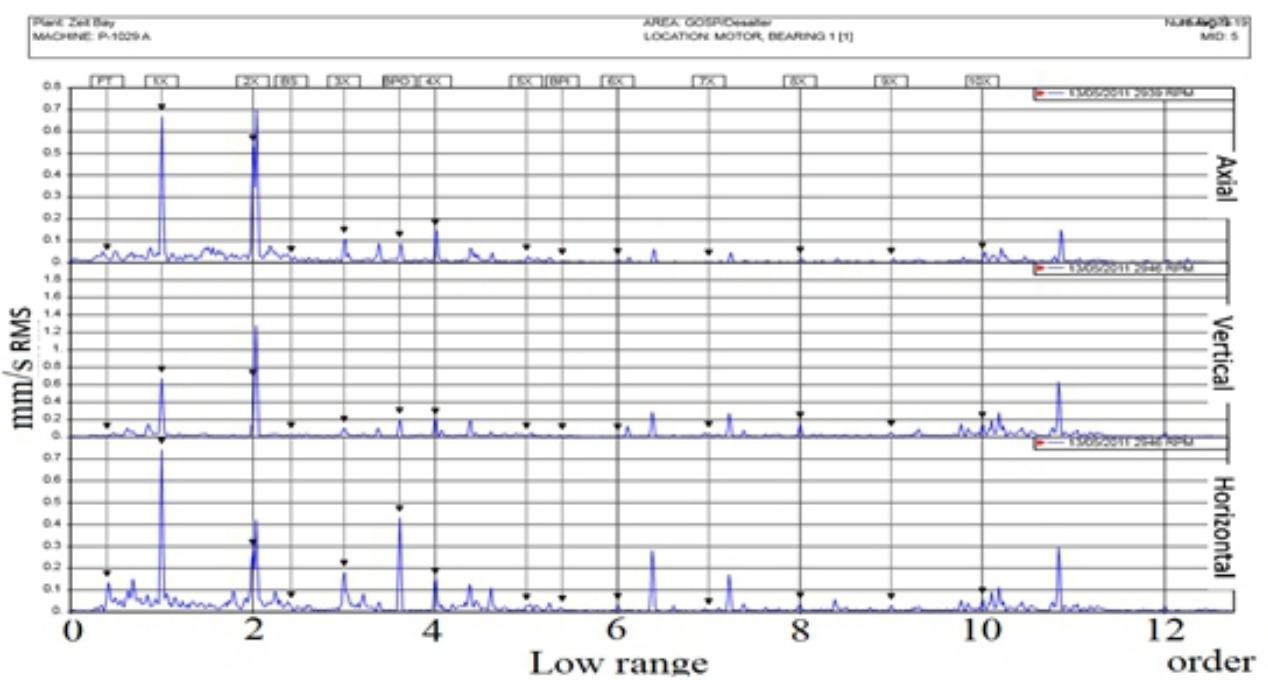

Figure 7. Spectrum of Motor NDE

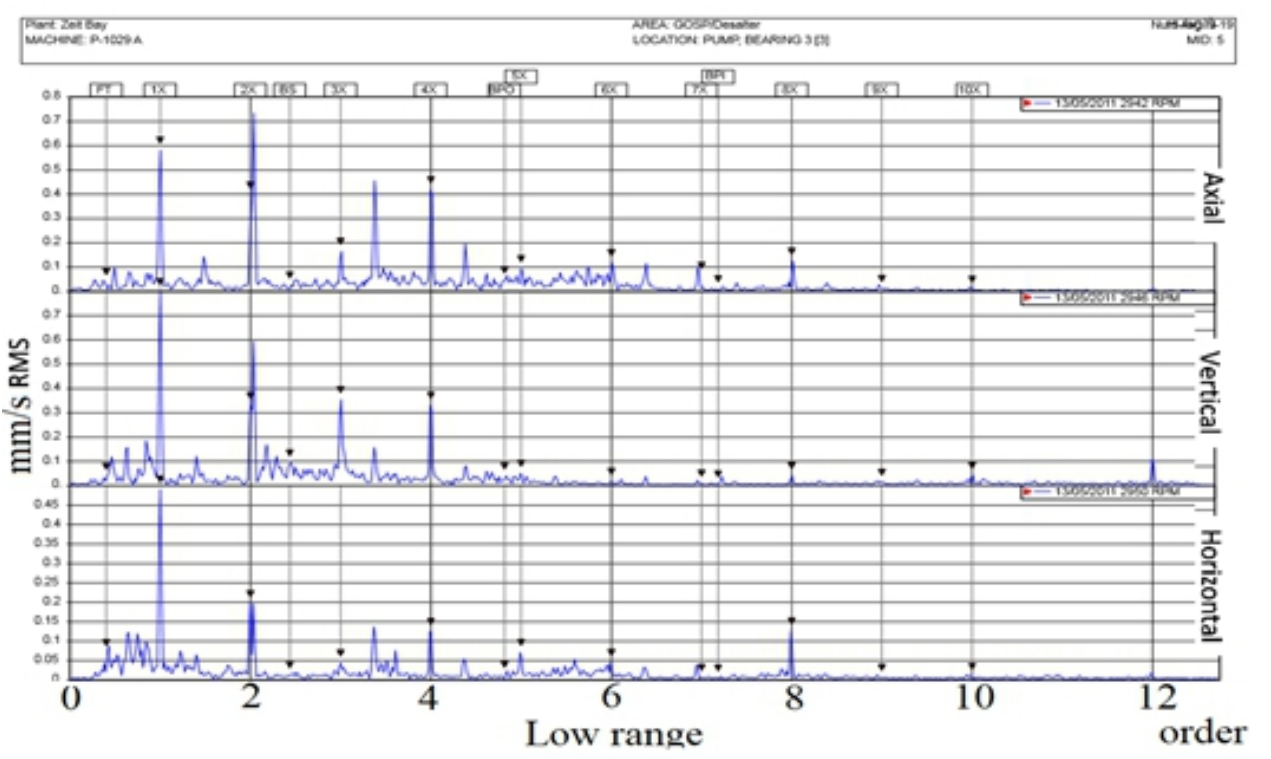

Figure 8. Spectrum of Pump DE

Figure 7 shows the spectrum of the motor. Also, we see that the maximum amplitude $(1.2 \mathrm{~mm} / \mathrm{s})$ in the vertical direction at $2 \mathrm{X}$ is the highest amplitude. As shown in this figure, the amplitude values of the axial, vertical, and horizontal at the FTF are $0.021,0.012$ and $0.06 \mathrm{~mm} / \mathrm{s}$, respectively. BSF are $0.007,0.036$ and $0.023 \mathrm{~mm} / \mathrm{s}, \mathrm{BPFO}$ are $0.088,0.204$ and $0.432 \mathrm{~mm} / \mathrm{s}$, and BPFI are $0.00908,0.016$ and $0.0139 \mathrm{~mm} / \mathrm{s}$, respectively. It is obvious that this case represents a normal case. Additionally, Figure 8 shows the spectrum of the pump DE. It is apparent that the maximum amplitude $(0.79 \mathrm{~mm} / \mathrm{s})$ in the 
vertical direction at $1 \mathrm{X}$ is the highest amplitude. As presented in this figure, the amplitude values of the axial, vertical, and horizontal at the FTF are $0.0290,0.0162$ and $0.0202 \mathrm{~mm} / \mathrm{s}, \mathrm{BSF}$ are $0.0086,0.0946$ and $0.0109 \mathrm{~mm} / \mathrm{s}$, BPFO are $0.0476,0.030$ and $0.032 \mathrm{~mm} / \mathrm{s}$, and BPFI are $0.0058,0.038$ and $0.0095 \mathrm{~mm} / \mathrm{s}$, respectively. It is observable that this case represents a normal case. Figure 9 shows the spectrum of the pump NDE. The maximum amplitude $(0.63 \mathrm{~mm} / \mathrm{s})$ in the vertical direction at $1 \mathrm{X}$ is the highest amplitude. As displayed in this figure, the amplitude values of the axial, vertical, and horizontal at the FTF are 0.0489, 0.0341 and $0.014 \mathrm{~mm} / \mathrm{s}, \mathrm{BSF}$ are $0.596,0.24$ and $0.215 \mathrm{~mm} / \mathrm{s}$, BPFO are $0.00747,0.0204$ and $0.025 \mathrm{~mm} / \mathrm{s}$, and BPFI are $0.0098,0.012$ and $0.0139 \mathrm{~mm} / \mathrm{s}$, respectively. It is noticeable that this case represents a normal case.

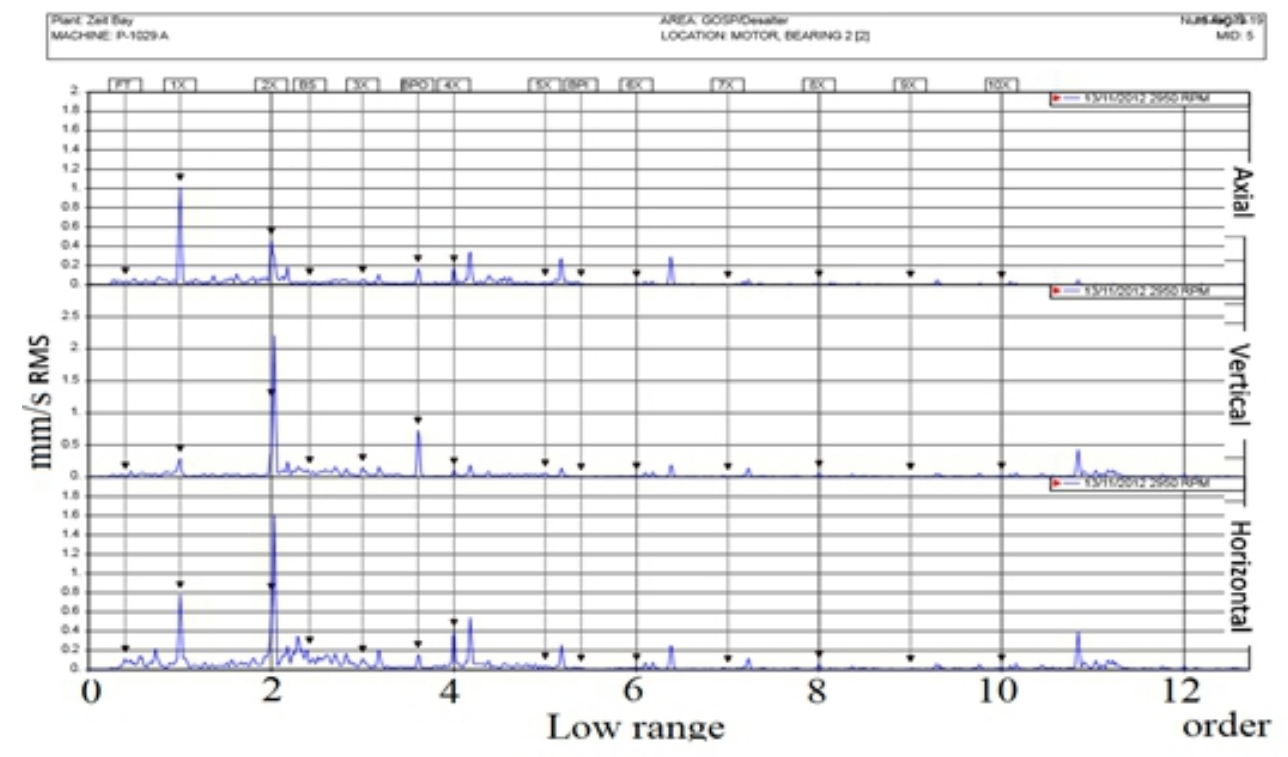

\section{Abnormal Operation}

Figure 9. Spectrum of Pump NDE

The frequency spectrum of the faulty pump was recorded, and Figure 10-13 show the frequency response in the faulty condition. Table 2 also shows the amplitude measurement readings at the abnormal operation at the four positions at the three axes. These readings are compared with the alarm and the critical limits. It is obvious that the readings are still under alarm levels.

Figure 10 shows the spectrum of the motor DE. The maximum amplitude $(2.2 \mathrm{~mm} / \mathrm{s})$ in the vertical direction at $2 \mathrm{X}$ is the highest amplitude. As shown in this figure, the amplitude values of the axial, vertical, and horizontal at the FTF are $0.034,0.029$ and $0.074 \mathrm{~mm} / \mathrm{s}$, BSF are $0.0305,0.055$ and $0.065 \mathrm{~mm} / \mathrm{s}$, BPFO are $0.169,0.73$ and $0.15 \mathrm{~mm} / \mathrm{s}$, and BPFI are 0.01106 , 0.00915 and $0.0129 \mathrm{~mm} / \mathrm{s}$, respectively. It is observed that this case represents a normal case. Furthermore, Figure 11 shows the spectrum of the motor NDE. 
The maximum amplitude $(2.56 \mathrm{~mm} / \mathrm{s})$ in the horizontal direction at $3.61 \mathrm{X}$ is the highest amplitude. As seen in this figure, the amplitude values of the axial, vertical, and horizontal at the FTF are 0.0138, 0.0145 and 0.0616 $\mathrm{mm} / \mathrm{s}, \mathrm{BSF}$ are $0.0232,0.0209$ and $0.0669 \mathrm{~mm} / \mathrm{s}$, BPFO are 0.328, 2.24 and $2.86 \mathrm{~mm} / \mathrm{s}$, and BPFI are $0.0151,0.0180$ and $0.0216 \mathrm{~mm} / \mathrm{s}$, respectively. It is important to note that there is a change in the BPFO values at vertical and horizontal directions. Figure 12 shows the spectrum of the pump DE. The maximum amplitude $(1.38 \mathrm{~mm} / \mathrm{s})$ in the axial direction at $4.17 \mathrm{X}$ is the highest amplitude which is normal. It is also noted that the amplitude values of the axial, vertical, and horizontal at the FTF are $0.046,0.082$ and $0.109 \mathrm{~mm} / \mathrm{s}$, $\mathrm{BSF}$ are $0.0166,0.0845$ and $0.0119 \mathrm{~mm} / \mathrm{s}, \mathrm{BPFO}$ are $0.103,0.038$ and 0.0308 $\mathrm{mm} / \mathrm{s}$, and BPFI are $0.053,0.0235$ and $0.050 \mathrm{~mm} / \mathrm{s}$, respectively. Figure 13 shows the spectrum of the pump NDE. The maximum amplitude $(3.05 \mathrm{~mm} / \mathrm{s})$ in the horizontal direction at 4.17X is the highest amplitude. Also, it is worthy to note that the amplitude value of the axial, vertical, and horizontal at the FTF are $0.0172,0.00242$ and $0.03719 \mathrm{~mm} / \mathrm{s}, \mathrm{BSF}$ are $0.59,0.313$ and $0.1005 \mathrm{~mm} / \mathrm{s}$, BPFO are $0.023,0.043$ and $0.0424 \mathrm{~mm} / \mathrm{s}$, and BPFI are $0.079,0.086$ and 0.046 $\mathrm{mm} / \mathrm{s}$, respectively.

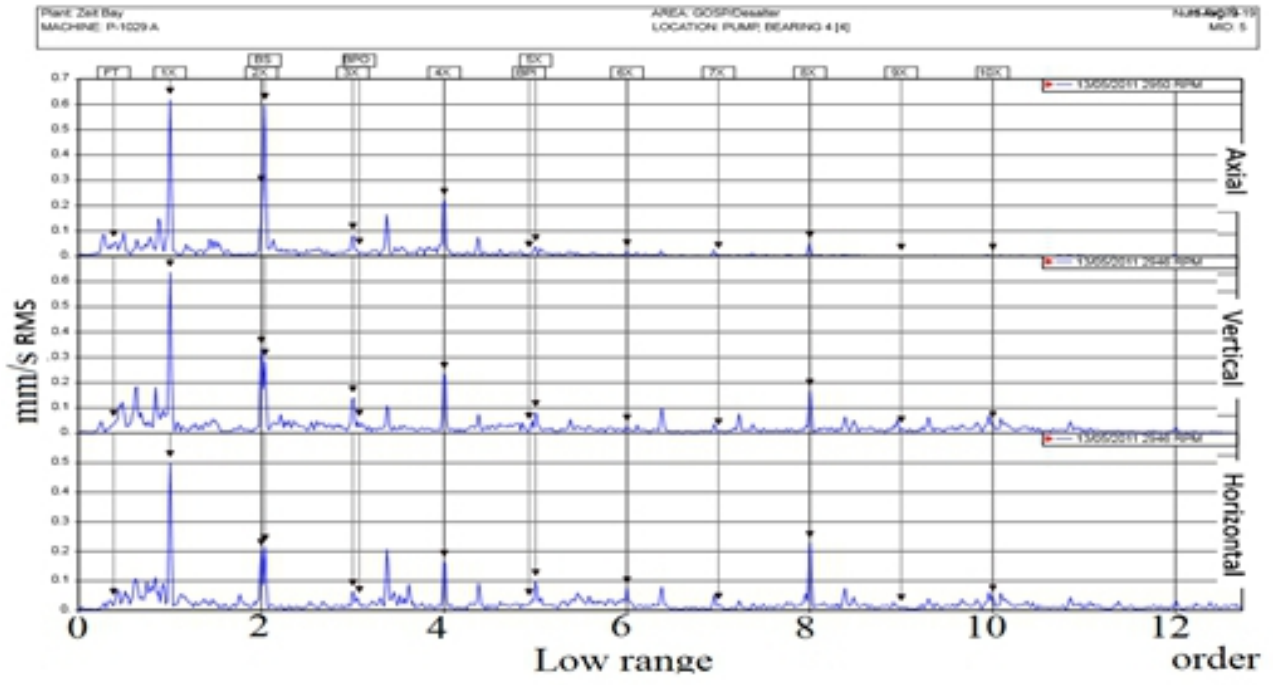

Figure 10. Spectrum of Motor DE 


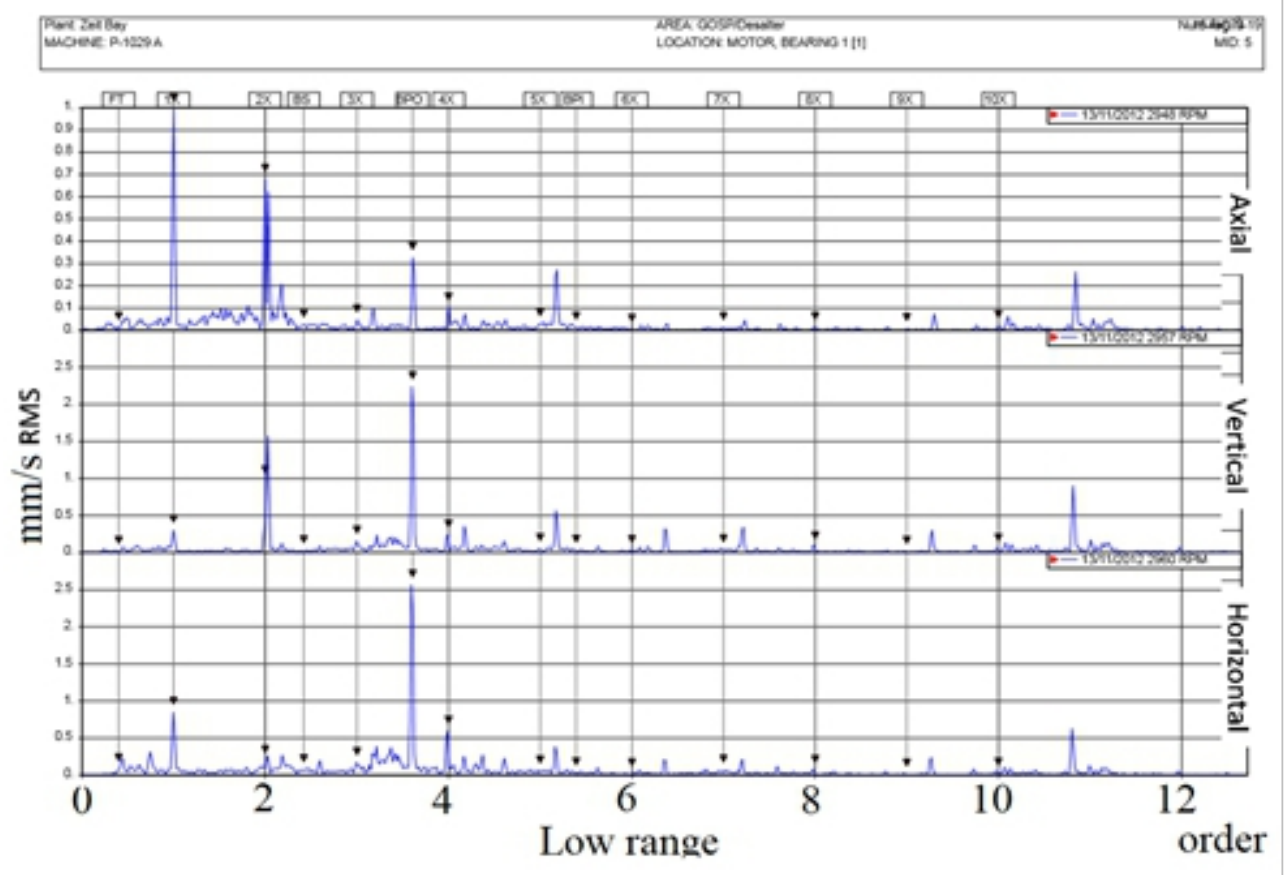

Figure 11. Spectrum of Motor NDE

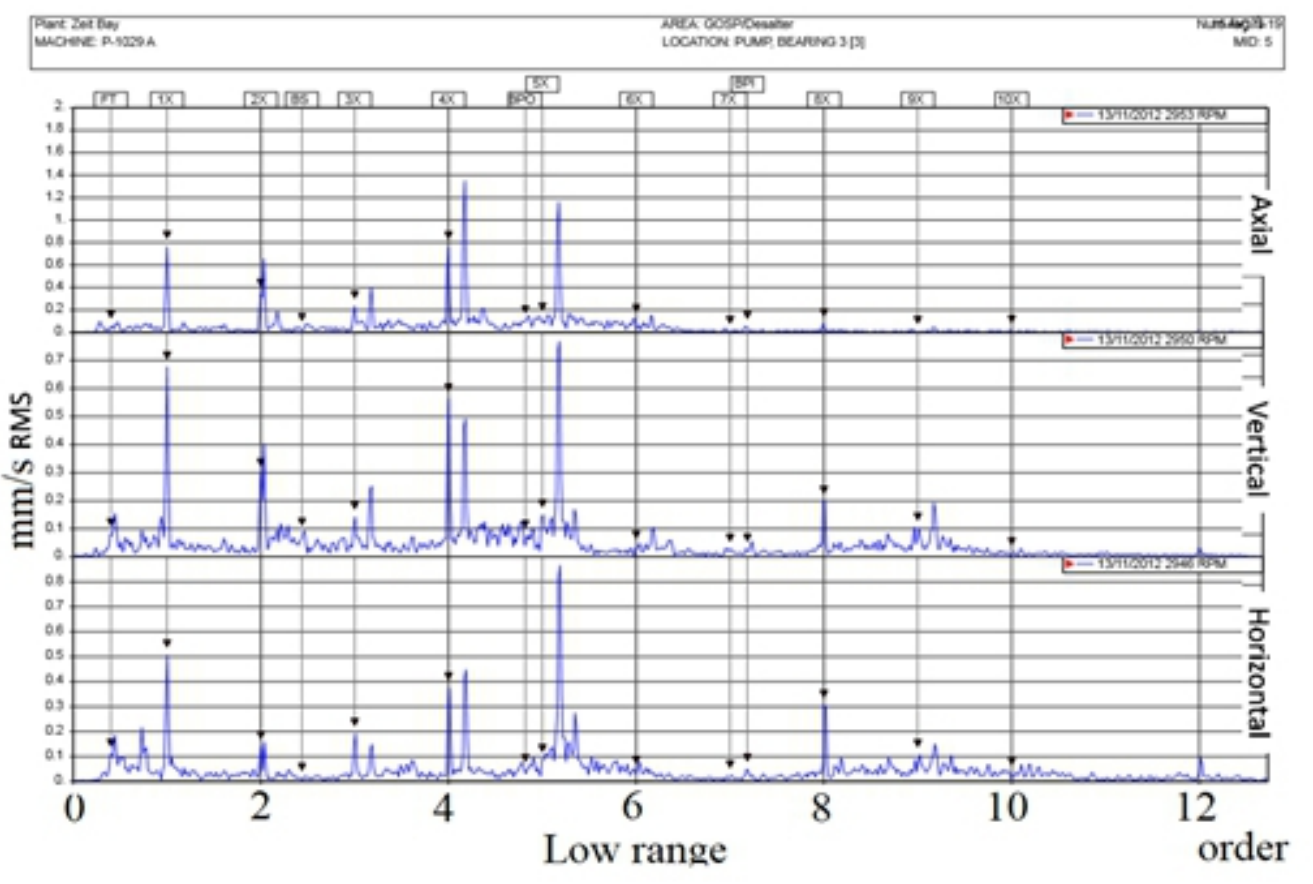

Figure 12. Spectrum of Pump DE 


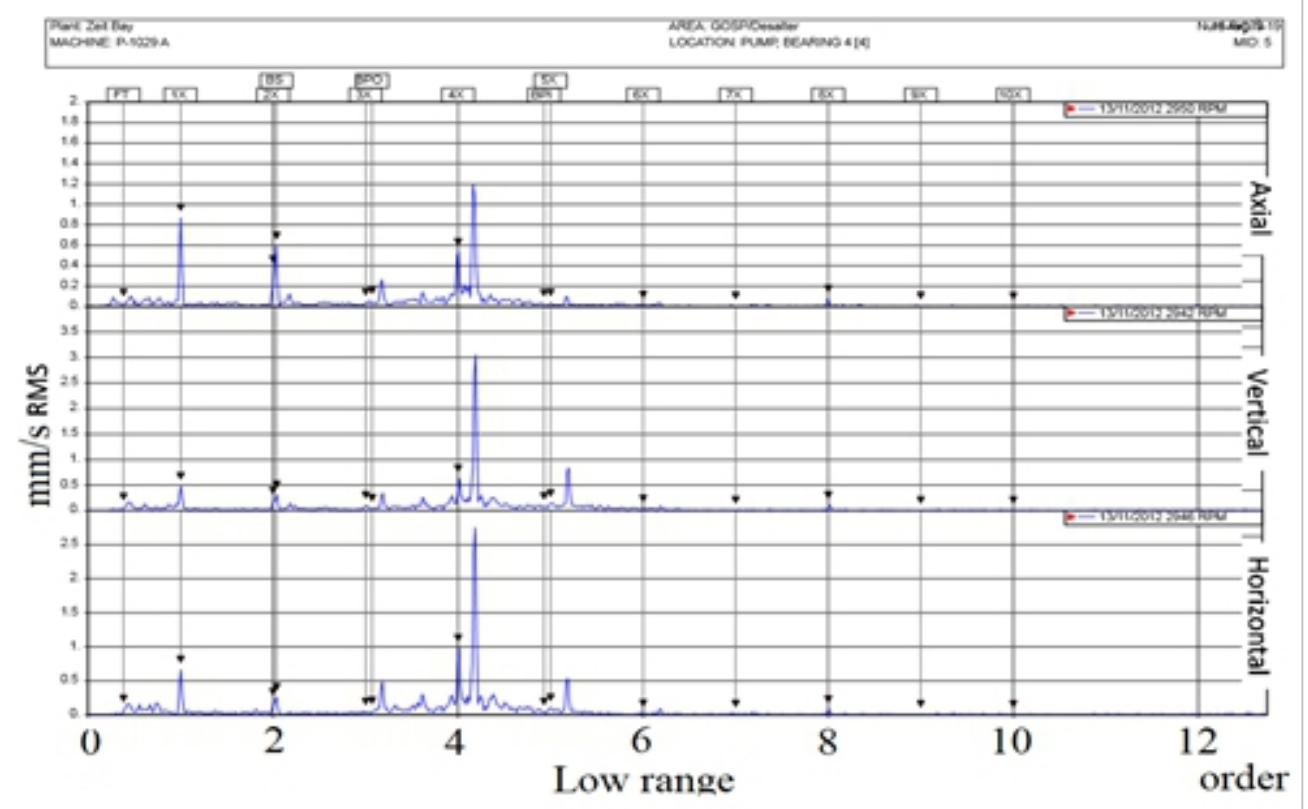

Figure 13. Spectrum of Pump NDE

Figure 14-17 show a comparison between the normal spectra (blue color) and abnormal spectra (red color) at the four positions. Figure 14 shows that there are slight changes observed in the vertical and horizontal direction at $2 \mathrm{X}$, but this change is not effective. Figure 15 shows that there is a change in vertical and horizontal direction at 3.61X BPFO frequency. Figure 16 also shows that there are slightly changes seen in the vertical and horizontal direction at $2 \mathrm{X}$, but this change is not effective. Figure 17 shows that the reading is very close, but there is a peak at frequency $4.17 \mathrm{X}$ with amplitude $3.05 \mathrm{~mm} / \mathrm{s}$ which appears at the faulty pump. 


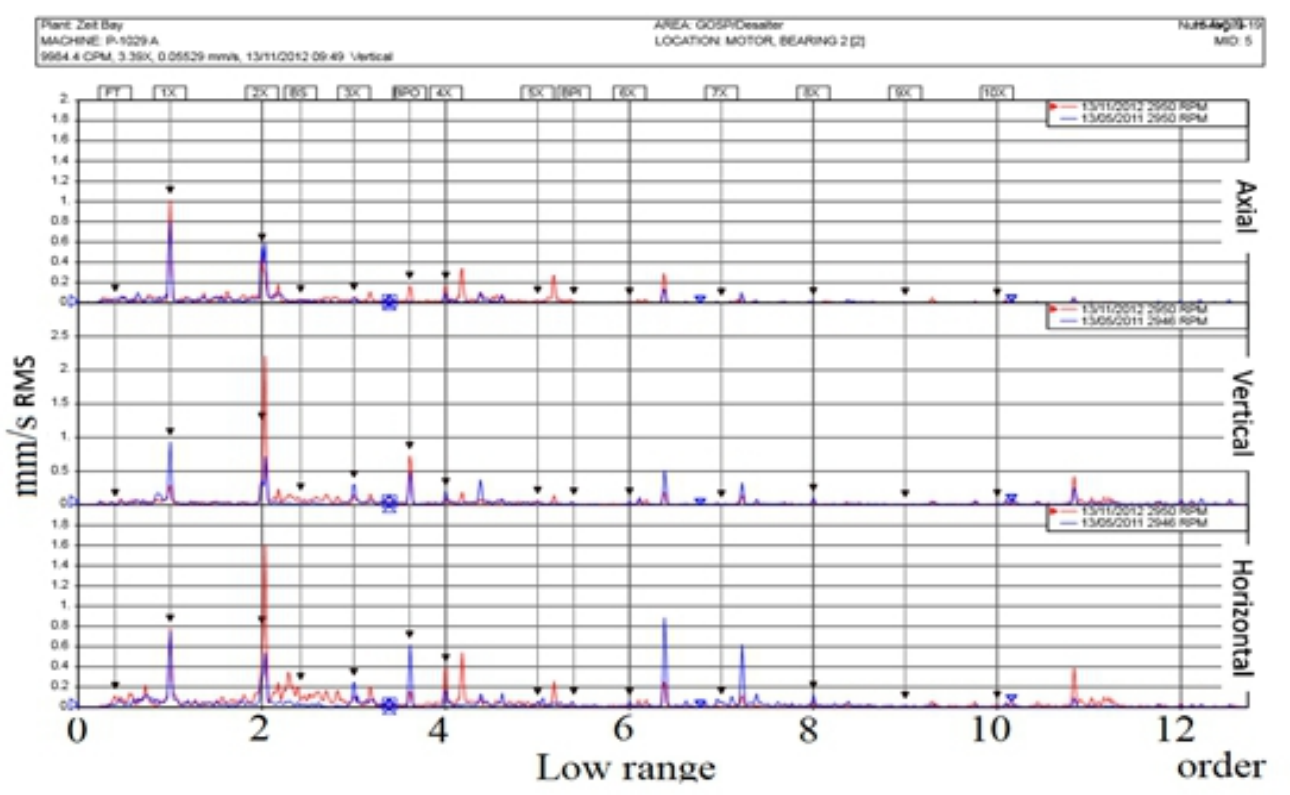

Figure 14. Comparison between Normal (blue color) and Abnormal (red color) Reading for Motor DE

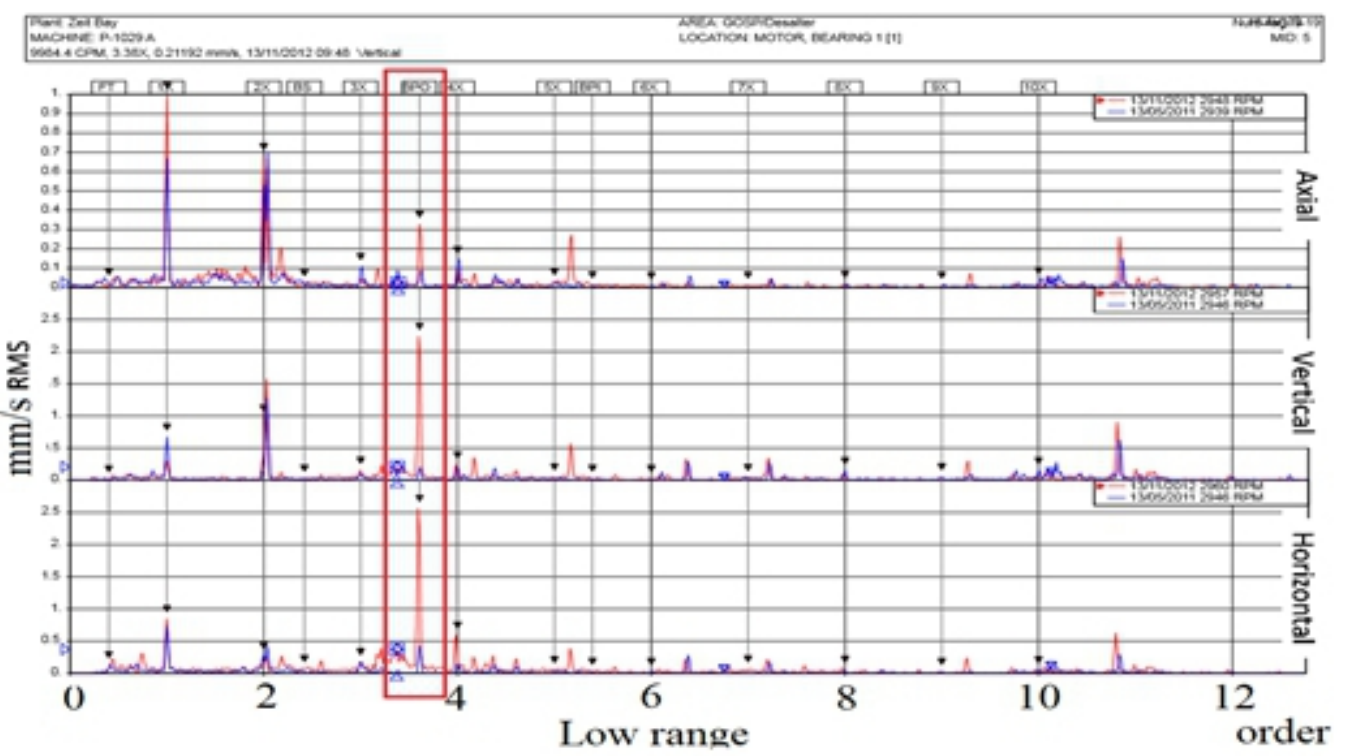

Figure 15. Spectrum Comparison between Normal (blue color) and Abnormal (red color) Reading for Motor NDE 


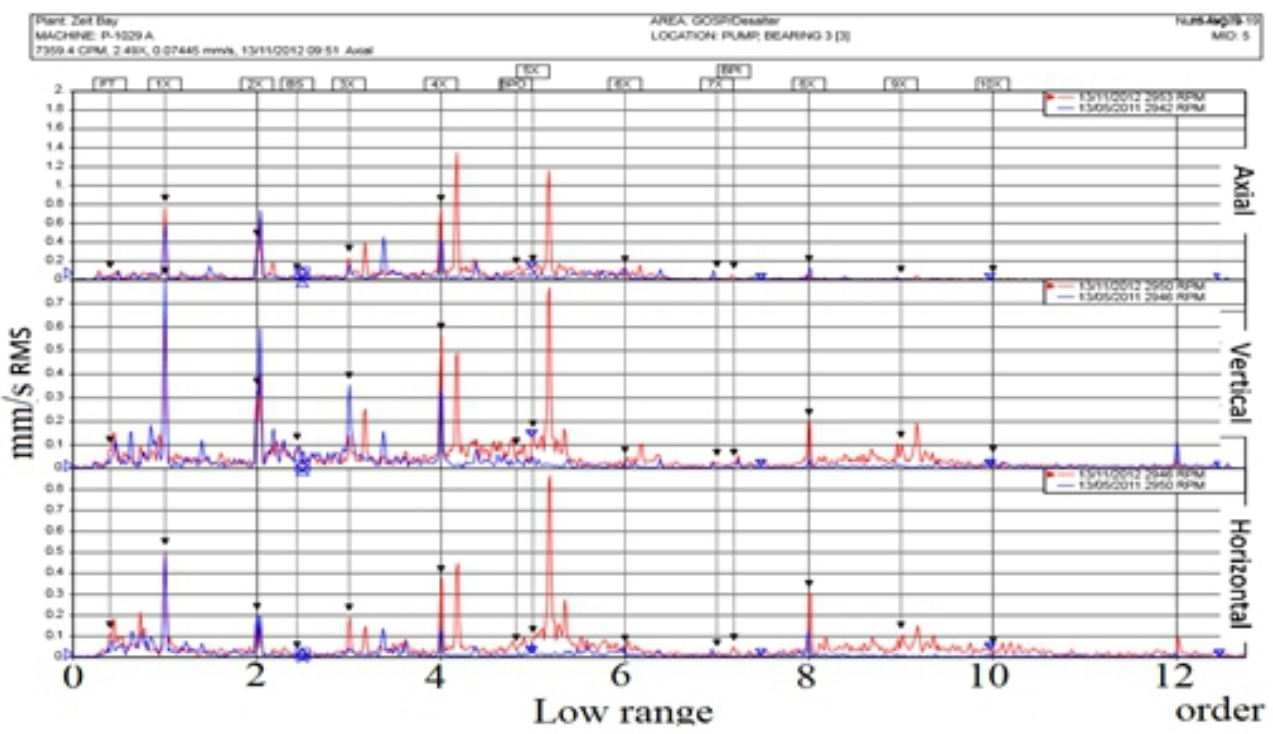

Figure 16. Spectrum Comparison between Normal (blue color) and Abnormal (red color) Reading for Pump DE

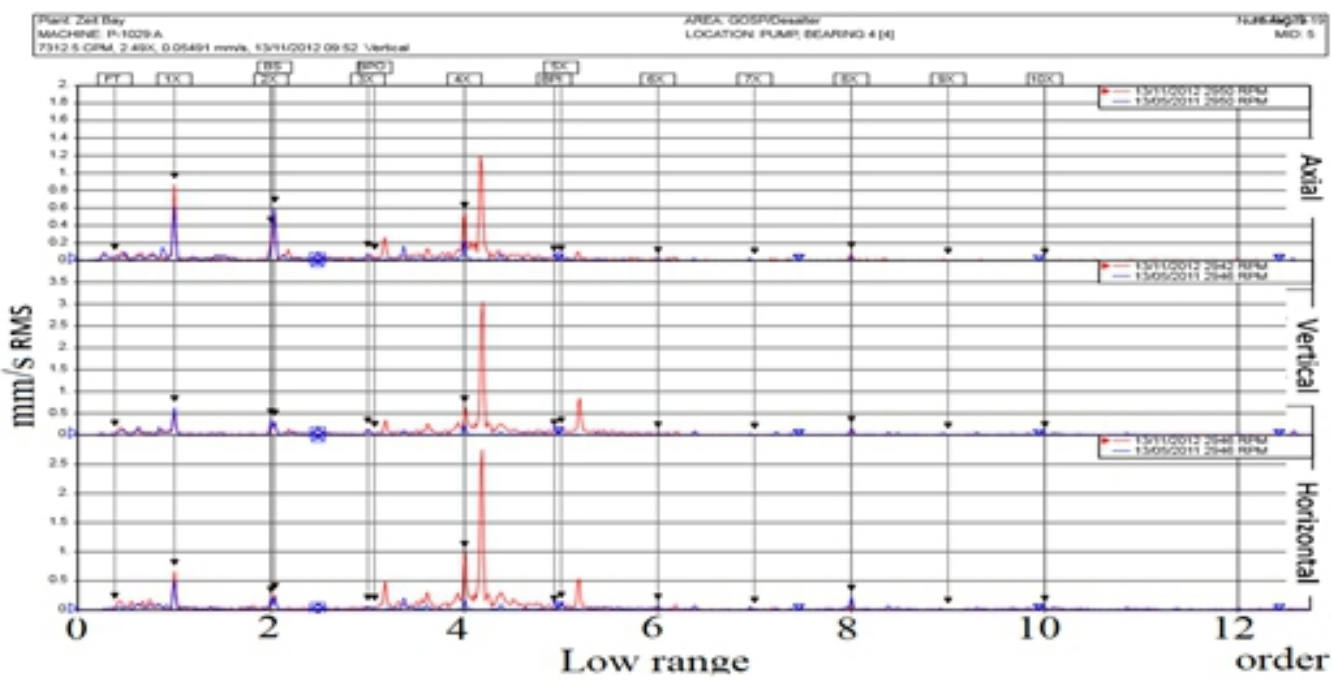

Figure 17. spectrum comparison between normal (blue color) and abnormal (red color) reading for pump NDE 


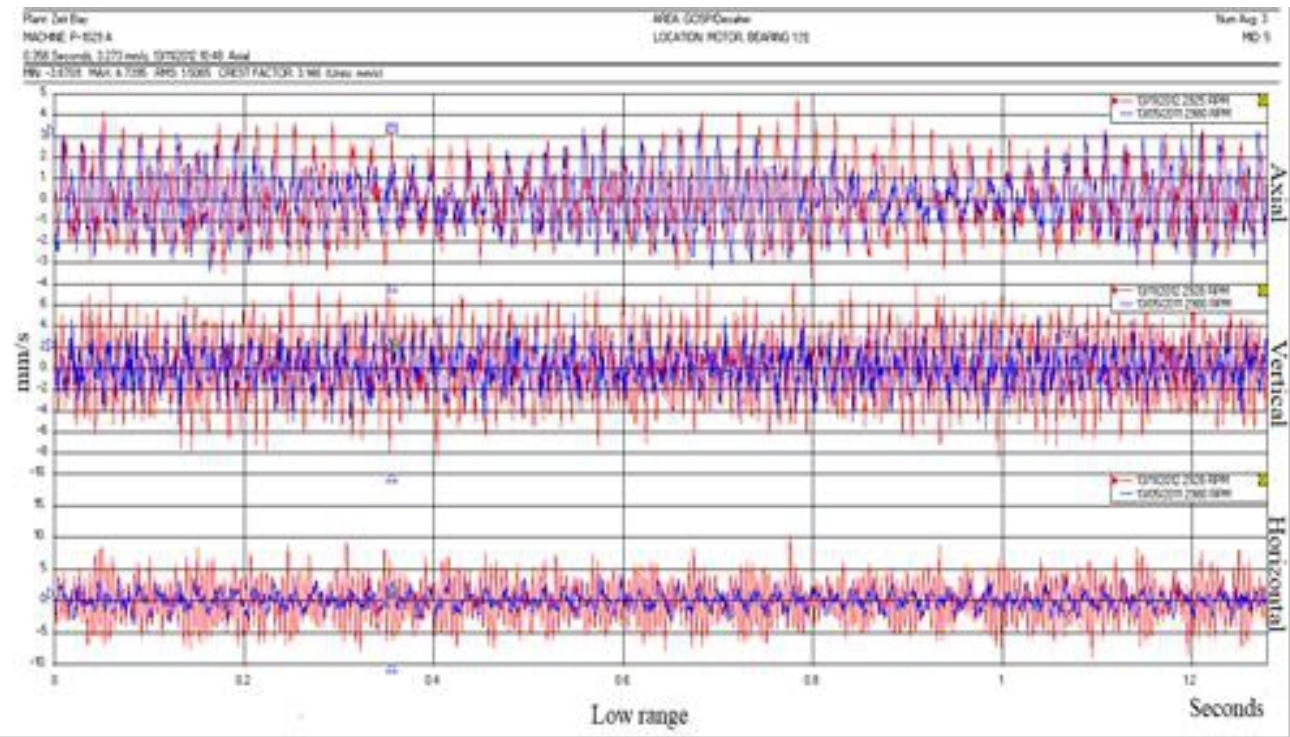

Figure 18. Time waveform comparison of motor NDE

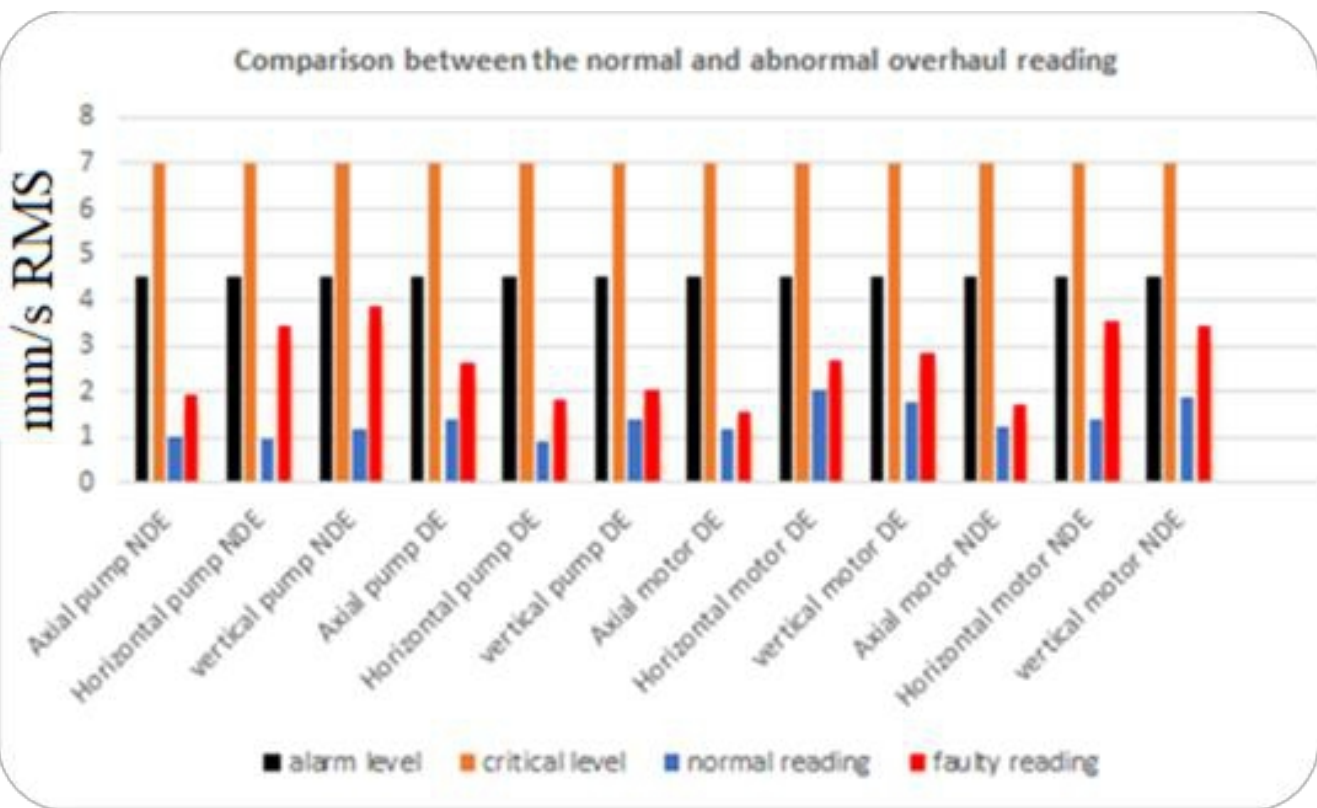

Figure 19. Comparison between the Normal and Abnormal Overall Reading

Vibration spectrum which compares the maximum average amplitudes between the normal and abnormal cases for all positions in three directions as shown in Figure 15-19 show the normal condition in "blue color" and the abnormal condition in "red color". The x-axis is in ordered scale, where the order " 1 " represents the rotational rate of the motor. The $y$-axis is in " $\mathrm{mm} / \mathrm{s}$ " scale for the amplitude after analyzing and comparing the spectrum of the three axes of the Motor NDE. We noted that the highest amplitudes peak was 
at the vertical and horizontal directions at a frequency of 3.61X. It appears that the amplitude of the normal readings was axial $=0.088 \mathrm{~mm}$, vertical $=0.204 \mathrm{~mm}$, and horizontal $=0.43 \mathrm{~mm}$. This became an abnormal reading at axial $=0.32 \mathrm{~mm}$, vertical $=2.24 \mathrm{~mm}$, and horizontal $=2.5 \mathrm{~mm}$. This frequency at $3.61 \mathrm{X}$ is identified as the ball pass frequency outer race (BPFO) of the SKF 6208 bearing. This change in the amplitude of the frequency (BPFO) indicates that the bearing is likely to be suffering from a wear problem and is entering stage 3 of bearing failure. It is recommended that the bearing should be replaced before the deterioration enters stage 4 or the catastrophic stage. Figure 18 shows the time waveform of the motor NDE and the pluses due to the impact at the PBFO frequency. Figure 19 shows the comparison between the alarm levels (black color), the critical levels (orange color), overall normal readings (blue color), and the abnormal readings (red color). It has obviously seen that the readings changed but did not exceed the alarm levels or the critical level. However, there is a difference between the normal readings on all the bearings. It also shows the abnormal readings compared with both normal and alarm level.

\section{Conclusion}

The remaining life of the bearings can be estimated using vibrational behavior and running time of the bearings. Due to the bearing wear, the failures of the bearing occur randomly, and the lubrication problem and the dynamic forces can be accelerated through this wear. Usually, the first discrete bearing frequency and the highest probability of wear happened at the inner race and the outer raceway. For the outer race, the progression of wear allows for trending where the load zone is relatively small with respect to the pitch diameter. Any change in the amplitude at the bearing component frequencies is considered an indication of the beginning of component failure.

\section{References:}

1. Amarnath, M., Shrinidhi, R., Ramchandra, A., \& Kandagal, S.B. (2004). "Prediction of defects in antifriction bearings using vibration signal analysis", Journal of the Institution of Engineers India, Vol. 85, PP. 88-92.

2. Brian Graney \& Ken Starry (2012). "Rolling element bearing analysis", Material Evaluation, Vol. 70(1), PP. 78-85.

3. (2012). "Vibration analysis for fault diagnosis of rolling element Ebrahim Ebrahimi bearings". Journal of American Science, Vol. 8(2), PP. 331-336.

4. ISO 10816-7 (2009). "Mechanical vibration -- Evaluation of machine vibration by measurements on non-rotating parts -- Part 7: Rotor 
dynamic pumps for industrial applications, including measurements on rotating shafts" International Organization for Standardization, part 7.

5. Matthew, J. \& Alfredson, R. J. (1984). "The condition monitoring of rolling element bearings using vibration analysis", Journal of Vibration, Acoustics, Stress, and Reliability in Design, Vol. 106(3), PP. 447-453.

6. Paresh Girdha \& Cornelius Scheffer (2004). "Practical machinery vibration analysis and predictive maintenance", IDC Technologies.

7. Ravindra A.Tarle, Nilesh K. Kharate, \& Shyam P. Mogal (2015). "Vibration analysis of ball bearing" International Journal of Science and Research (IJSR), Vol. 4(5), PP. 2655-2665.

8. Shinde Ashaykumar, Desavale Ramchandra, \& Kumbhar Surajkumar (2014). "Theoretical and experimental studies on vibrations produced by defects in double row ball bearing using response surface method", International Journal of Research in Engineering and Technology, Vol.3(5), PP. 140-145

9. Sharma, S., Kankar, P.K., \& Agrawal, V.P (2011). "Fault identification in rolling bearing using vibration signature analysis "Depart of Mechanical Engineer, Thapar University, Patiala (Punjab).

10. Tandon, N. \& Choudhary, A. (1997). "An analytical model for the prediction of the vibration response of rolling element bearings due to a localized defect", Journal of Sound and Vibration, Vol. 205 (3), PP. 275-292.

11. Tandon, N. \& Choudhary, A. (1998). "A theoretical model to predict vibration response of rolling bearings to distributed defects under radial load" Journal of Tribology-Transactions of the ASME, Vol. 120(1), PP. 214-220.

12. Zeki Kiral \& Hira Karagulle (2005). "Vibration analysis of rolling element bearings with various defects under the action of an unbalanced force ", Mechanical Systems and Signal Processing, Vol. 20(8), PP. 1967-1991. 
Appendix (ISO 10816) [12]

\begin{tabular}{|c|c|c|c|c|c|c|}
\hline \multicolumn{7}{|c|}{ VIBRATION SEVERITY PER ISO 10816} \\
\hline \multicolumn{3}{|c|}{ Machine } & \multirow{2}{*}{$\begin{array}{l}\text { Class I } \\
\text { small } \\
\text { machines }\end{array}$} & \multirow{2}{*}{$\begin{array}{l}\text { Class II } \\
\text { medium } \\
\text { machines }\end{array}$} & \multirow{2}{*}{$\begin{array}{l}\text { Class III } \\
\text { large rigid } \\
\text { foundation }\end{array}$} & \multirow{2}{*}{$\begin{array}{l}\text { Class IV } \\
\text { large soft } \\
\text { foundation }\end{array}$} \\
\hline & $\mathrm{in} / \mathrm{s}$ & $\mathrm{mm} / \mathrm{s}$ & & & & \\
\hline \multirow{12}{*}{ 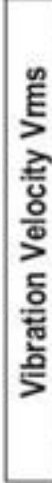 } & 0.01 & 0.28 & & & & \\
\hline & 0.02 & 0.45 & & & & \\
\hline & 0.03 & 0.71 & & \multicolumn{2}{|c|}{ good } & \\
\hline & 0.04 & 1.12 & & & & \\
\hline & 0.07 & 1.80 & & & & \\
\hline & 0.11 & 2.80 & & \multicolumn{2}{|c|}{ satisfactory } & \\
\hline & 0.18 & 4.50 & & & & \\
\hline & 0.28 & 7.10 & & \multicolumn{2}{|c|}{ unsatisfactory } & \\
\hline & 0.44 & 11.2 & & & & \\
\hline & 0.70 & 18.0 & & & & \\
\hline & 0.71 & 28.0 & & \multicolumn{2}{|c|}{ unacceptable } & \\
\hline & 1.10 & 45.0 & & & & \\
\hline
\end{tabular}

\section{Abbreviation}

\section{A Axial}

BPFI Bearing Pass Frequency-Inner Race

BPFO Bearing Pass Frequency Outer Race

BSF Bearing Spin Frequency (Balls)

CPM Cycle Per Minute

DE Drive End

DSS Deflected Shaped Shaft

FFT Fast Fourier Transformation

FTF Fundamental Train Frequency Cage

$\mathrm{H}$ Horizontal

HFD High Frequency Detection

HP High Pressure $\theta \quad$ Contact Angle in Degrees

MDE Motor Drive End

MNDE Motor Non-Drive End

NDE Non-Drive End

PDE Pump Drive End

PNDE Pump Non-Drive End

RMS Root Mean Square

rpm revolution per minute

V Vertical

Pd Bearing Pitch Diameter

$\mathrm{N}_{b} \quad$ Number of Balls or Rollers

$1 \mathrm{X} 1 \mathrm{st}$ order of the frequency 